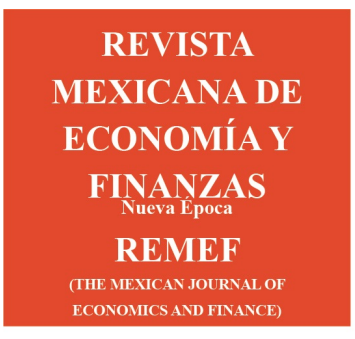
Revista Mexicana de Economía y Finanzas Nueva Época
Volumen 15 Segundo Número Especial Aniversario Agosto $2020 \quad$ pp. $523-536$
DOI: https://doi.org/10.21919/remef .v15i0.544
(Recibido: 13/abril/2020, aceptado: 21/agosto/2020)

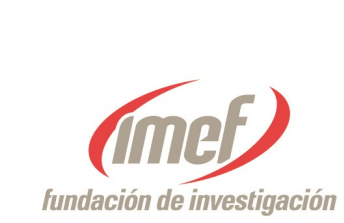

SNEA - Entrepreneurial research in emerging economies: recent advances Editor Invitado: Dr. Samuel Mongrut

\title{
Evaluación integral del ecosistema emprendedor de Costa Rica y propuestas para su mejora
}

\author{
Catalina Núñez-Álvarez ${ }^{1}$ \\ Asesora Independiente en Virtualización Educativa, Costa Rica \\ Juan Carlos Leiva ${ }^{2}$ \\ Instituto Tecnológico de Costa Rica, Costa Rica
}

\section{Resumen}

El objetivo de este artículo fue evaluar el estado del ecosistema emprendedor en Costa Rica al segundo semestre de 2018. La metodología consistió en una conjugación de cinco modelos diferentes de valoración de clasificación de ecosistemas emprendedores. Esos cinco modelos juntos contienen sesenta y seis variables, las cuales fueron armonizadas mediante ocho grandes categorías. Los resultados indican que el ecosistema emprendedor costarricense posee en términos generales un estado regular (2,03 en escala de 1 a 5$)$, destacándose algunos aspectos negativos como el financiamiento y la investigación y desarrollo, así como otros medianamente positivos como la infraestructura. El trabajo posee como limitación que los datos disponibles en cada modelo son de diferentes momentos en el tiempo. Los resultados tienen implicaciones relacionadas con diversas oportunidades de mejora para el ecosistema emprendedor costarricense en lo referente a la coordinación de sus actores y la generación de acciones para fortalecer los componentes más débiles.

Clasificación JEL: L26, M13, O38, H50, I23, D21

Palabras clave: Ecosistema Emprendedor, Emprendimiento Dinámico, Emprendimiento Costarricense

\section{Integral Assessment of the Entrepreneurial Ecosystem of Costa Rica and Improvement Proposals}

\section{Abstract}

The purpose of this paper was to evaluate the Costa Rican entrepreneurial ecosystem at the 2018 second semester. The methodology employed a conjugation of five different models for assessing entrepreneurial ecosystems. Those five models contain sixty-six variables, which were harmonized by eight broad categories. The results indicate that the Costa Rican entrepreneurial ecosystem has in general terms a regular condition (2.03 on a scale of 1 to 5), highlighting some negative aspects such as financing and research and development, as well as other moderately positive such as infrastructure. The work has the limitation that the data available in each model are from different moments in time. The results have implications related to various opportunities for improvement for the Costa Rican entrepreneurial ecosystem in relation to the coordination of its actors and the generation of actions to strengthen the weakest components.

\footnotetext{
${ }^{1}$ Correo: hablemos@catalinanunez.com

${ }^{2}$ Escuela de Administración de Empresas, Instituto Tecnológico de Costa Rica. Campus Cartago, Costa Rica. Orcid: https://orcid.org/0000-0001-9653-4629

*Sin fuente de financieamiento para el desarrollo de la investigación
} 


\section{Abstract}

JEL Classification: L26, M13, O38, H50, I23, D21

Keywords: Entrepreneurial Ecosystem, Dynamic Entrepreneurship, Costa Rican Entrepreneurship

\section{Introducción}

El tema del emprendimiento es relevante para la sociedad. La evidencia científica indica que su efecto contribuye con el desarrollo de las naciones; sin embargo, es un fenómeno complejo de estudiar, a causa de que es sistémico y posee múltiples aristas. (Szerb, et al, 2018).

En los últimos diez años, Costa Rica ha contado con tres políticas públicas en materia de emprendimiento emitidas por el Ministerio de Economía, Industria y Comercio (MEIC): (1) "Costa Rica Emprende", publicada en diciembre de 2010 como parte de la Administración Chinchilla-Miranda 2010-2014 (MEIC, 2010); (2) "Política de Fomento al Emprendimiento en Costa Rica 2014-2018", en el siguiente gobierno, el de la Administración Solís-Rivera (MEIC, 2014) y la Política Nacional de Empresariedad 2030 en la presente administración Alvarado Quesada 2018-2022 (MEIC, 2020).

Estas políticas han producido una serie de iniciativas que se espera reditúen en un mejor contexto para el emprendimiento, o dicho en términos académicos, eleve la calidad del ecosistema emprendedor costarricense, entendiendo un ecosistema en este ámbito como la forma mediante la cual diversos elementos de carácter social, material y cultural se combinan en formas complejas, para propiciar u obstaculizar, el surgimiento y desarrollo de nuevas empresas (Isenberg, 2010; Spigel 2017).

Esta visión de ecosistema es, en la actualidad, la más utilizada para analizar el surgimiento de nuevas empresas y su posterior desarrollo. Varias circunstancias contribuyen a ello. En primer lugar, hay abundante evidencia científica sobre su impacto en la competitividad de las regiones o países (Acz et al., 2018). Incluso hay evidencia de cómo el mismo ecosistema emprendedor posee un rol moderador entre los factores; observándose incluso que, aún, cuando las políticas públicas se inclinen a incrementar la cantidad de emprendimientos, esto no tendrá efecto positivo en la economía si no se articula con un ecosistema emprendedor sano (Szerb et al., 2018).

No obstante, al ser un campo emergente, existen aún muchas preguntas sin responder. Inclusive no existe una definición comúnmente aceptada del concepto. La literatura al respecto puede categorizarse en dos grandes perspectivas. Una primera, generada por académicos principalmente, que se orienta a definir cuáles son los componentes que debería tener un ecosistema emprendedor (Brown \& Mason 2017). Por otro lado, en la segunda línea, desde una perspectiva de practitioners la literatura busca medir, valorar y ubicar en un ranking los ecosistemas emprendedores de las naciones (Spigel \& Harrison 2018).

Dentro de esa segunda perspectiva hallamos cinco modelos o metodologías que evalúan y comparan los ecosistemas emprendedores de diversos países (Kantis, et al., 2017; Ács, et al., 2018; Lebendiker, et.al., 2015; Cornell University, INSEAD \& World Intellectual Property Organization, 2018; World Bank Group, 2018).

Nuestro trabajo tuvo como objetivo evaluar el estado del Ecosistema Emprendedor de Costa Rica (EECR) al segundo semestre de 2018 mediante una metodología que permitiera armonizar los cinco modelos hallados ${ }^{3}$. El análisis incluye además un estudio comparativo con otros ecosistemas de referencia para el costarricense.

\footnotetext{
${ }^{3}$ Nuestro artículo se limita exclusivamente al análisis de los modelos de practitioners, excluyendo los de índole académica (Brown \& Mason 2017). Dado que es un análisis trasversal tampoco abarcamos temas como la mejoría o en su caso el deterioro de los ecosistemas como un ente abierto. Para estos abordajes sugerimos revisar trabajos como los de McKenzie \& Sud, 2009; O'Connor et al., 2018; Stam, 2018).
} 
Los resultados abarcan una valoración integral del ecosistema emprendedor costarricense contemplando una relación armonizada entre las variables de los cinco modelos identificados, así como un listado de oportunidades de mejora, cubriendo temas como los retos de financiamiento, la articulación entre actores, vacíos en el mercado, sostenibilidad e innovación.

Lo que resta del trabajo se organiza de la siguiente forma. En primer lugar, se muestra el fundamento teórico del estudio. Posteriormente se explica la metodología que se siguió para el desarrollo del trabajo. La siguiente sección muestra los resultados, incluyendo una aplicación práctica para Costa Rica, así como las oportunidades de mejora detectadas. Finalmente, el artículo cierra con las conclusiones, limitaciones y futuras investigaciones sugeridas.

\section{Marco Teórico}

La primera mención sobre la metáfora biológica de ecosistemas para comprender el fenómeno emprendedor se atribuye a James Moore en 1993, no obstante, fue Daniel Isenberg quien popularizó el concepto en el mundo académico ya entrado el siglo XXI (Brown \& Manson, 2017). Dicho autor propuso que la política, los mercados, el financiamiento, el capital humano, la cultura y los apoyos "se combinan en formas complejas", ya que cada elemento propicia el emprendimiento, sin embargo, es juntos como propician de forma efectiva la creación y crecimiento de las empresas (Isenberg, 2010). La figura 1 presenta la estructura de Isenberg.

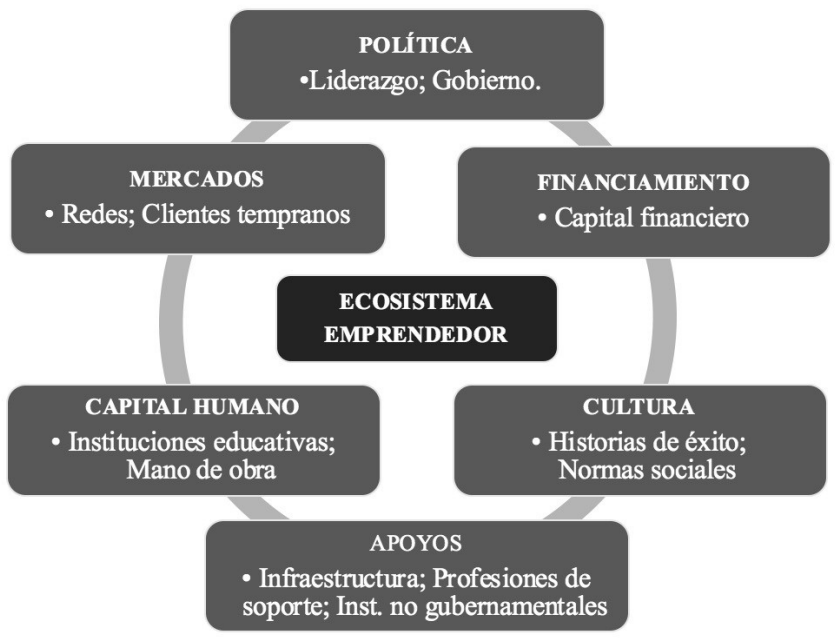

Figura 1. Nivel 1 y 2 de los dominios del Ecosistema Emprendedor según Isenberg.

Después de Isenberg, diversos autores han hecho sus aportaciones en el tema de ecosistema emprendedor. $\mathrm{Al}$ respecto, nuestro argumento teórico es que han existido al menos dos grandes líneas de análisis. En una de ellas se ha generado una discusión, desde la perspectiva teórica, sobre cuáles son los componentes que debería tener un ecosistema emprendedor (Feld 2012; Qian et al.,2013; Mason \& Brown 2014; Auerswald 2015; Stam 2015; Audretsch \& Belitski 2016; Spigel 2017; Brown \& Mason 2017). Por otro lado, en la segunda línea, desde una perspectiva de practitioners los esfuerzos se han dirigido a medir, valorar y ubicar en un ranking los ecosistemas emprendedores de las naciones (Spigel \& Harrison 2018).

En este sentido las propuestas buscan generar índices y poder efectuar análisis comparativos o de mejores prácticas (Kantis, et al.,2017, Ács, et al.,2018; Lebendiker, et al.,2015; Cornell University, INSEAD \& World Intellectual Property Organization, 2018; World Bank Group, 2018). El aporte de nuestro trabajo es tratar de hacer un ejercicio de unificación de los trabajos más consistentes dentro de la segunda perspectiva de análisis y aplicarlo al análisis de un estudio de caso como el de Costa Rica. 
En la primera perspectiva, hay varios trabajos que pueden ilustrar el estilo de abordaje. Por ejemplo, Stam (2015) propone diez componentes: redes (network), liderazgo, financiamiento, talento, conocimiento, servicios de apoyo/intermediarios, instituciones formales, cultura, infraestructura física, y demanda. Su trabajo es teórico y se fundamenta en una síntesis de diversos modelos que han sido publicados en diversas revistas científicas. Por su lado, Spigel (2017) propone tres grandes categorías (culturales, sociales, materiales) para agrupar diez variables en su propuesta de ecosistema emprendedor: soporte cultural, historias de emprendimiento, talento laboral, capital inversionista, redes de contactos, mentores y modelos de rol, política y gobernanza, universidades, servicios de apoyo, infraestructura física, y mercados abiertos. En su trabajo utiliza dos casos de estudio en Canadá para ilustrar el concepto propuesto.

En la segunda perspectiva de análisis, tal y como se mencionaba, se pueden ver esfuerzos por medir, valorar y ubicar en un ranking los ecosistemas emprendedores de las naciones. En esta perspectiva hemos detectado dos proyectos que buscan medir de forma integral la totalidad del ecosistema emprendedor (Kantis et al, 2017; Acs et al. 2018), uno que busca medir la actividad emprendedora de las personas (Global Entrepreneurship Research Association 2018) y dos que miden otros temas pero que abarcan algunos específicos que atañen al tema de emprendimiento (Cornell University, INSEAD \& World Intellectual Property Organization, 2018; World Bank Group, 2018).

El trabajo pionero en la temática ha sido el Global Entrepreneurship Monitor (GEM), el cual desde 1999 ha emitido sus informes para analizar la actividad emprendedora de las personas, tal y como se mencionó previamente. Su enfoque es valorar las actitudes y conductas emprendedoras de las personas en un país por medio de encuestas directas a una muestra representativa de personas. Adicionalmente valora el entorno o ecosistema, pero bajo un enfoque cualitativo de entrevistas con expertos.

Por otro lado, Kantis y colegas desde el año 2014 emiten su informe anual titulado "Índice de Condiciones Sistémicas para el Emprendimiento Dinámico" (ICSED) el cual ha venido evolucionando para llamarse en 2018 "The Index of Dynamic Entrepreneurship". Este índice pone el foco de atención en emprendimientos dinámicos y bajo su metodología el ecosistema emprendedor se compone de diez dimensiones: capital humano emprendedor, cultura, educación, condiciones sociales, estructura empresarial, condiciones de la demanda, plataforma de ciencia y tecnología, capital social, financiamiento, regulaciones/políticas. (Kantis et al., 2019).

En la misma línea de acción, pero con otras variables, Acs et al., (2019) desde 2009 proponen el Global Entrepreneurship Index (GEI) un modelo de ecosistema emprendedor que abarca 14 pilares: percepción de oportunidades, habilidades para la creación de empresas startup, tolerancia al riesgo, redes (networking), soporte cultural, oportunidades para nuevas empresas startup, absorción de tecnología, capital humano, presencia de competencia, innovación de productos, innovación de procesos, crecimiento acelerado de empresas, internacionalización, capital de riesgo.

Los otros dos informes referenciados analizan otros temas, innovación en el caso del Global Innovation Index (GII) (Cornell University, INSEAD \& World Intellectual Property Organization, 2018) y la facilidad de hacer negocios en el Doing Business (World Bank Group, 2018). En ambos casos existen temas específicos que se incluyeron para el análisis del ecosistema emprendedor acá propuesto, como el acceso al financiamiento, así como a infraestructura.

Para la integración de los modelos se buscó seguir una metodología validada, seleccionándose la de Aspen Network of Development Entrepreneurs (ANDE, 2013), la cual consta de ocho determinantes: (1) Financiamiento, (2) Soporte Empresarial, (3) Política, (4) Mercados, (5) Capital Humano, (6) Infraestructura, (7) Investigación \& Desarrollo (I+D) y (8) Cultura Emprendedora. Bajo este grupo de determinantes se buscó unificar los resultados de los cinco modelos previamente seleccionados. En la tabla 1 se puede ver la definición conceptual de las variables que servirán como agrupadoras de las otras de los cinco modelos analizados. 
Tabla 1. Definición conceptual de los determinantes.

\begin{tabular}{|c|c|}
\hline Nombre de la variable & Definición conceptual \\
\hline Financiamiento & $\begin{array}{l}\text { Disponibilidad de recursos financieros, capital y deuda; de } \\
\text { parte de individuos e inversores institucionales; incluidos: } \\
\text { bancos, ángeles, inversión privada, bolsa y filantropía. }\end{array}$ \\
\hline Soporte Empresarial & $\begin{array}{l}\text { Programas de todos los niveles de gobierno que ayudan a } \\
\text { las empresas nuevas y en crecimiento }{ }^{2} \text {. Considera la calidad } \\
\text { y cantidad de servicios legales, contables, de incubación } \\
\text { (instituciones que promueven la creación de negocios y su } \\
\text { crecimiento). También tiene que ver con cuán conectados y } \\
\text { cercanos están los emprendedores entre sí. }\end{array}$ \\
\hline Política & $\begin{array}{l}\text { Medida en que las políticas de gobierno apoyan a las nuevas } \\
\text { empresas y no afectan a las existentes y establecidas; entre } \\
\text { otras: tasas, incentivos, ambiente de negocios, nivel de burocracia } \\
\text { (costos y tiempo); también cuánto los emprendedores pueden } \\
\text { percibir oportunidades y el gobierno facilita que las aprovechen. }\end{array}$ \\
\hline Mercados & $\begin{array}{l}\text { Medida en que las prácticas comerciales habituales y el marco } \\
\text { regulatorio son flexibles de forma que las empresas (todas) puedan } \\
\text { competir e incluso reemplazar proveedores; contempla las ventas } \\
\text { domésticas, internacionales y el tamaño del mercado y cuánto los } \\
\text { emprendedores crean nuevos productos para participar en el mercado } \\
\text { doméstico e internacional. }\end{array}$ \\
\hline Capital Humano & $\begin{array}{l}\text { Cuán altamente educados están los emprendedores, capacitados en } \\
\text { negocios y con facilidad de moverse en el mercado laboral. Involucra } \\
\text { el esfuerzo educativo (colegios, universidades y otros) para desarrollar } \\
\text { destrezas, habilidades y conocimiento para crear o dirigir negocios. }\end{array}$ \\
\hline Infraestructura & $\begin{array}{l}\text { Acceso a recursos físicos como transporte, telecomunicaciones, } \\
\text { electricidad, infraestructura física; con precios y facilidad que no } \\
\text { discrimine ni desfavorezca especialmente a las empresas micro, } \\
\text { pequeñas y nuevas. }\end{array}$ \\
\hline Investigación y Desarrollo (I+D) & $\begin{array}{l}\text { También tiene que ver con la capacidad de desarrollar nuevos } \\
\text { productos e integrarlos con tecnología, así como innovar procesos. } \\
\text { Toma en cuenta la cantidad de patentes generadas y acuerdos tecnológicos. } \\
\text { Lo cual conduce a generar nuevas oportunidades comerciales y cuánto } \\
\text { están al alcance de las empresas nuevas, micros y en crecimiento. }\end{array}$ \\
\hline Cultura Emprendedora & $\begin{array}{l}\text { Medida en que las normas culturales y sociales alientan acciones } \\
\text { individuales a llevar una nueva manera de conducir negocios o actividades } \\
\text { económicas; se relaciona con cómo un país ve el emprendimiento, la } \\
\text { proclividad al riesgo, la narrativa de los medios respecto a emprendimiento. }\end{array}$ \\
\hline
\end{tabular}

Fuente: elaboración propia.

\section{Metodología}

\subsection{Datos y variables}

La investigación se basó en los cinco modelos localizados en la revisión literaria, desde un enfoque de practitioners, con información disponible para el segundo semestre del año 2018, a saber: ICSED, GEI, GEM, GII, y Doing Business. Tal y como se mencionó, dado que las variables definidas en los cinco modelos base del estudio son diferentes, fueron agrupadas en las categorías del ANDE (2013) según se verá adelante. 
Es importante señalar que la propuesta de ANDE (2013) está basada en una evaluación de nueve marcos; entre ellos emitidos por Babson College, Global Entrepreneurship and Development Institute, World Economic Forum, World Bank y el modelo del Organismo para la Cooperación y el Desarrollo Económicos (OCDE). Además, ANDE se alinea más a la estructura original de Isenberg, por lo cual tiene la ventaja de que destaca elementos como soporte empresarial e infraestructura.

\subsection{Método de evaluación}

El primer paso consistió en analizar cada modelo por separado. Posteriormente el segundo paso fue establecer las ocho definiciones operacionales que pueden verse en la tabla 2, donde se indican los componentes con los cuales fueron medidos dichos determinantes en cada uno de los modelos internacionales contemplados.

Tabla 2. Definición operacional de los determinantes

\begin{tabular}{|c|c|}
\hline Nombre de la variable & Definición operacional \\
\hline Ecosistema Emprendedor & $\begin{array}{l}\text { Medido principalmente según los modelos ICSES 2017, GEI 2018, } \\
\text { GEM Costa Rica 2014, GEM 2017/18; adicionalmente con GII } \\
\text { 2018, Doing Business 2018. }\end{array}$ \\
\hline Financiamiento & $\begin{array}{l}\text { Financiamiento (ICSED 2017); Capital de riesgo (GEI 2018); } \\
\text { Entorno financiero relacionado con el emprendimiento (GEM } \\
\text { 2014, GEM 2017/18); Crédito, Inversión (GII 2018). }\end{array}$ \\
\hline Soporte Empresarial & $\begin{array}{l}\text { Estructura empresarial (ICSED 2017); Networking (GEI 2018); } \\
\text { Programas gubernamentales, Acceso a infraestructura comercial } \\
\text { y profesional (GEM 2014, GEM 2017/18). }\end{array}$ \\
\hline Política & $\begin{array}{l}\text { Políticas y regulaciones (ICSED 2017);Percepción de oportunidad, } \\
\text { Inicio de oportunidad (GEI 2018); Políticas gubernamentales } \\
\text { concretas, apoyo y prioridad, Políticas gubernamentales, burocracia } \\
\text { y tasas (GEM 2014, GEM 2017/18); Ambiente político, Ambiente } \\
\text { regulatorio, Ambiente de negocios (GII 2018); Comenzar un negocio, } \\
\text { Tratar con permisos de construcción, Registrar una propiedad, } \\
\text { Conseguir crédito, Proteger a inversores minoritarios, Pagar } \\
\text { impuestos, Comercializar afuera de las fronteras, Contratos de } \\
\text { refuerzo, Resolver insolvencias (Doing Business 2018). }\end{array}$ \\
\hline Mercados & $\begin{array}{l}\text { Condiciones de la demanda (ICSED 2017); Competencia, } \\
\text { Internacionalización, Alto Crecimiento (GEI 2018); Mercadointerno: } \\
\text { Dinámica, Mercado interno: Barreras (GEM 2014, GEM 2017/18); } \\
\text { Comercio, competencia y escala del mercado (GII 2018). }\end{array}$ \\
\hline Capital Humano & $\begin{array}{l}\text { Capital humano emprendedor, Educación, Condiciones sociales, } \\
\text { Capital social (ICSED 2017); Habilidades de arranque (startup), } \\
\text { Capital humano, Absorción de tecnología (GEI 2018); Nivel de } \\
\text { emprendimiento en la educación primaria y secundaria, Nivel de } \\
\text { emprendimiento con la educación vocacional (GEM 2014, GEM } \\
\text { 2017/18); Educación, Educación terciaria, Trabajadores del conocimiento, } \\
\text { Enlaces de innovación, Absorción de conocimiento (GII 2018). }\end{array}$ \\
\hline Infraestructura & $\begin{array}{l}\text { Acceso a infraestructura física y servicios (GEM 2014, GEM 2017/18); } \\
\text { Tecnologías de la información y comunicación, Infraestructura general, } \\
\text { Sustentabilidad ecológica (GII 2018); Conseguir electricidad (Doing } \\
\text { Business 2018). }\end{array}$ \\
\hline Investigación y Desarrollo $(\mathrm{I}+\mathrm{D})$ & $\begin{array}{l}\text { Plataforma de Ciencia y Tecnología para la Innovación (ICSED 2017); } \\
\text { Innovación de producto, Innovación de proceso (GEI 2018); Nivel de } \\
\text { transferencia de I+D (GEM 2014, GEM 2017/18); Salidas de conocimiento } \\
\text { y tecnología, Salidas creativas (GII 2018). }\end{array}$ \\
\hline Cultura Emprendedora & $\begin{array}{l}\text { Condiciones culturales (ICSED 2017); Aceptación al riesgo, Apoyo } \\
\text { cultural (GEI 2018); Normas sociales y culturales (GEM 2014, GEM 2017/18) }\end{array}$ \\
\hline
\end{tabular}

Fuente: elaboración propia. 
El tercer paso fue construir una matriz (tabla) en la cual se utilicen estos 8 determinantes a modo de categorías, dentro de las cuales se pueden distribuir las sesenta y siete variables que suman los cinco modelos. Para lograr la clasificación de cada variable en el determinante idóneo, fue consultada minuciosamente la información metodológica de cada modelo, así como los componentes que ANDE sugiere para el diagnóstico de cada determinante del octeto. A modo de ilustración y por limitación de espacio, la figura 2 muestra solamente una parte de esta matriz resultante; específicamente la distribución de los determinantes Financiamiento y Soporte Empresarial.

\begin{tabular}{|c|c|c|c|c|c|c|}
\hline \multirow[b]{2}{*}{ Determinantes } & \multicolumn{3}{|c|}{ ANÁLISIS PRINCIPAL } & \multicolumn{2}{|c|}{ ANÁLISIS ADICIONAL } & \multirow{2}{*}{$\begin{array}{c}\text { TEÓRICO } \\
\text { E.E Around } \\
\text { The Globe } \\
(\ldots)\end{array}$} \\
\hline & $\begin{array}{l}\text { ICSedProdem } \\
2018\end{array}$ & GEI 2018 & $\begin{array}{c}\text { GEM Costa } \\
\text { Rica } 2014\end{array}$ & GII 2018 & \begin{tabular}{|c|} 
Doing \\
Business 2018
\end{tabular} & \\
\hline Financiamiento & Financiamiento. & $\begin{array}{l}\text { Capital de } \\
\text { riesgo. }\end{array}$ & $\begin{array}{l}\text { Entorno } \\
\text { financiero } \\
\text { relacionado con } \\
\text { el } \\
\text { emprendimiento. }\end{array}$ & $\begin{array}{l}\text { Crédito. } \\
\text { Inversión. }\end{array}$ & & $\begin{array}{l}\text { Fondos y } \\
\text { financiamiento }\end{array}$ \\
\hline $\begin{array}{c}\text { Soporte } \\
\text { Empresarial }\end{array}$ & $\begin{array}{l}\text { Estructura } \\
\text { empresarial } \\
\text { (Eje2). }\end{array}$ & Networking. & $\begin{array}{l}\text { Programas } \\
\text { gubernamentales. } \\
\text { Acceso a } \\
\text { infraestructura } \\
\text { comercial y } \\
\text { profesional. }\end{array}$ & & & $\begin{array}{l}\text { Sistema de } \\
\text { apoyo / } \\
\text { mentores. }\end{array}$ \\
\hline
\end{tabular}

Figura 2. Representación parcial de la matriz de los 67 componentes Fuente: elaboración propia.

El cuarto paso del proceso consistió en diseñar una herramienta en Excel para procesar la información de todos los modelos. Este libro, cuya hoja de índice puede observarse en la figura 3, contiene varias tablas y gráficos automatizados.

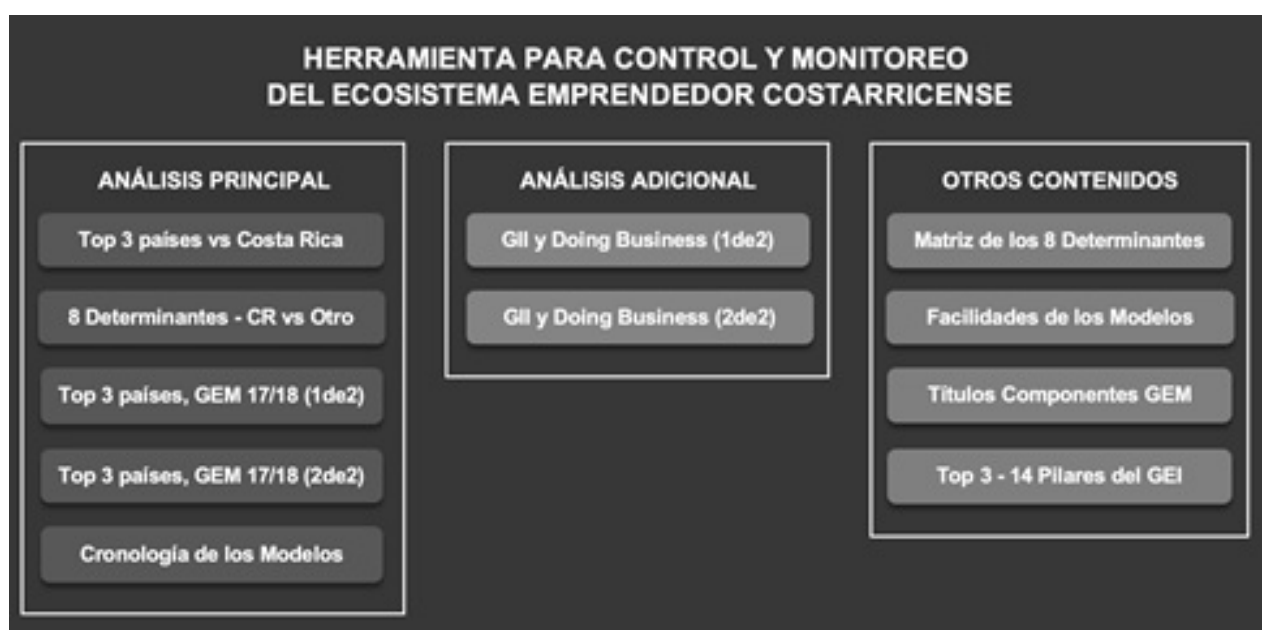

Figura 3. Índice de herramienta diseñada para el análisis

Fuente: elaboración propia. 
El principal, es la interfaz que contiene 8 tablas, que corresponden cada una a un determinante de la matriz de clasificación. La figura 4 muestra a manera de ejemplo la tabla del determinante Soporte Empresarial. Cada tabla agrupa las variables de dicho determinante. Como un detalle aclaratorio, cabe mencionar que los determinantes no agrupan necesariamente la misma cantidad de variables, lo que sí conserva cada tabla es la estructura para anotar y nivelar el puntaje.

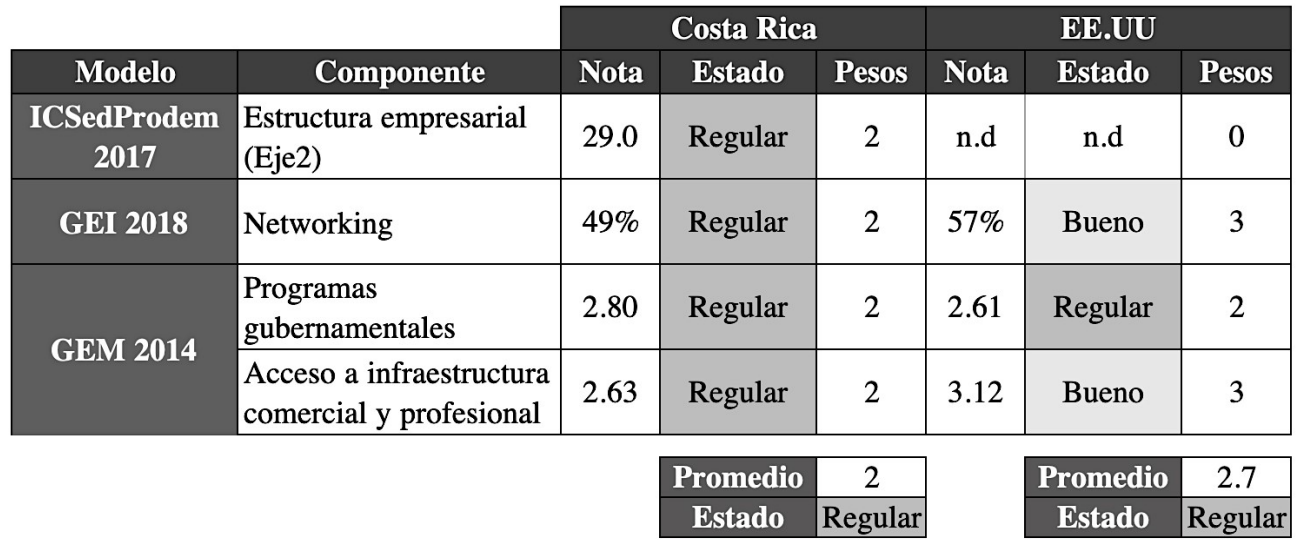

Figura 4. Tablas de componentes: determinante de Soporte Empresarial

Fuente: elaboración propia.

Esta nivelación de puntaje se refiere a un sistema de semáforo construido para esta metodología, el cual estandariza las notas de cada componente. La necesidad para utilizarlo radica en que los modelos seleccionados presentaban diferentes rangos de calificación. Tanto ICSED 2017 como el GII califican de 0 a 100, GEI 2018 y Doing Business 2018 califican de 0 a $100 \%$; sin embargo, el GEM Costa Rica 2014 utiliza una escala Likert de 1 a 9 . De tal manera que el semáforo diseñado permite estandarizar de 0 a 5 , o bien de deficiente a excelente, ya sea las escalas de 0 a 100 o la de 1 a 9 . Permitiendo generar resultados promedio de estado de cada determinante y finalmente construir una tabla resumen para estimar el estado de todo el Ecosistema Emprendedor. Resultados que serán presentados adelante en este artículo.

La herramienta construida también incluye, entre otros elementos, tablas y gráficos para presentar los tres mejores países de los modelos, una cronología del puesto obtenido por Costa Rica en los diferentes modelos desde 2006, el análisis comparativo de los modelos de innovación (GII) y facilidad de hacer negocios (Doing Business).

Finalmente, el quinto paso de nuestra metodología consistió en el análisis de los resultados. Es importante mencionar que, para efectos del artículo, se analizó la situación de Costa Rica como nación y además de manera comparativa con dos países adicionales, Estados Unidos y México. Esta selección, a modo de benchmarking, responde a dos criterios. En el caso de Estados Unidos dado que lidera el ranking en el GEI 2018 y ostenta el segundo puesto en el ICSed-Prodem 2017. En el caso de México se busca aportar una comparación latinoamericana, y este país se ubicó un puesto arriba que Costa Rica en el ICSED 2017. Además, en ambos casos Costa Rica posee abundante cercanía geográfica, cultural y de negocios con dichos países. La figura 5 presenta un resumen gráfico de la metodología de análisis seguida. 


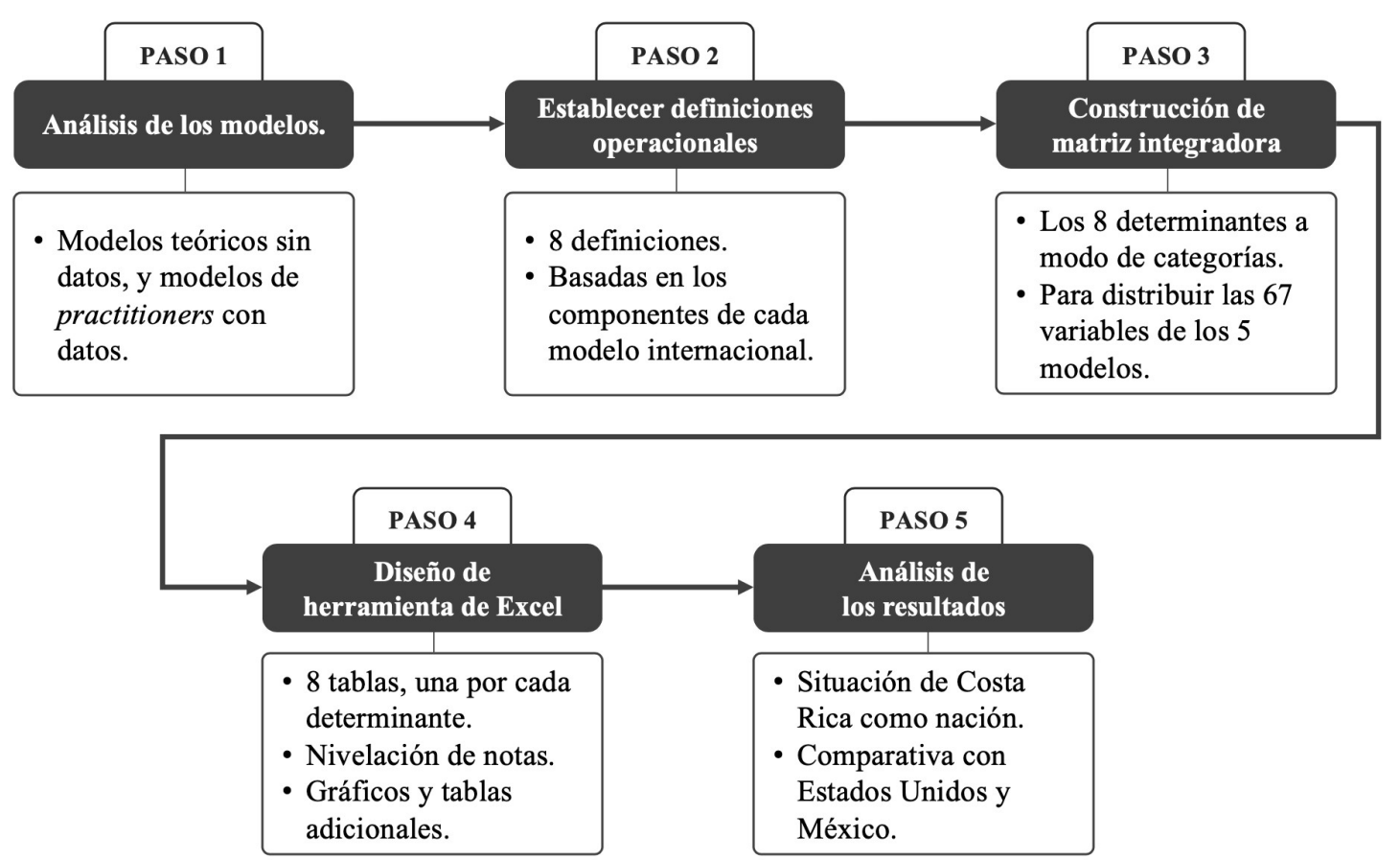

Figura 5. Paso a paso: elaboración de metodología propuesta Fuente: elaboración propia.

\section{Resultados}

\subsection{Aplicación práctica para Costa Rica}

El puesto obtenido por Costa Rica en los modelos evaluados, es representado en la figura 6 (en orden: 43, $56,54,61$ ) respecto al total de países contemplados (dato en el borde inferior de cada columna). Esta figura deja notar la brecha entre el puesto de esta nación centroamericana y el puesto número uno. Es importante notar la relatividad del puesto de Costa Rica, ya que, a manera de ejemplo, para el ICSED y el GEI Costa Rica obtuvo notas muy similares (32,97 y 33,3\% respectivamente), sin embargo, su posición varía en trece puestos debido a que cada ranking incorpora diferentes países y la posición de cada nación se verá afectada tanto por su nota como la de los demás integrantes del estudio.

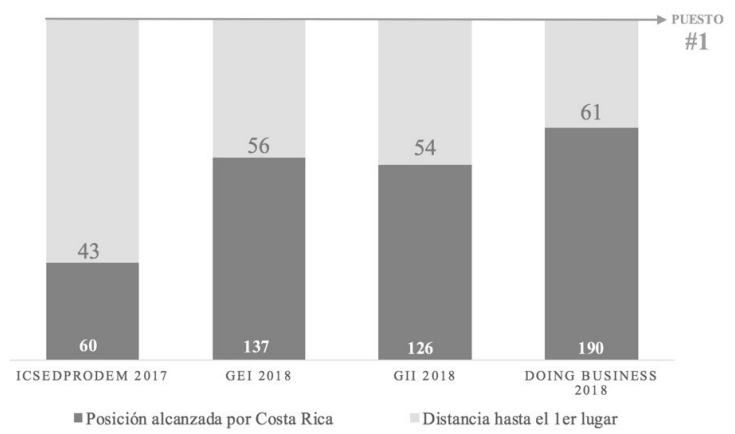

Figura 6. Brecha entre puesto obtenido por Costa Rica y el primer lugar de cada índice Fuente: elaboración propia.

Nota: El GEM no genera puestos a manera de ranking, razón por la cual no está incluido en la figura 3. 
Según fue señalado, Estados Unidos (EE.UU) y México fueron usados como referentes para el análisis. La Figura 7 muestra el resultado final luego de evaluar individualmente cada determinante mediante el ICSED 2017, GEI 2018 y GEM 2014.

\begin{tabular}{|c|c|c|c|c|}
\hline \multicolumn{2}{|c|}{ Determinante } & Costa Rica & México & EE.UU \\
\hline 1 & Financiamiento & Deficiente & Deficiente & Regular \\
\hline 2 & Soporte Empresarial & Regular & Regular & Regular \\
\hline 3 & Política & Regular & Regular & Bueno \\
\hline 4 & Mercados & Regular & Deficiente & Excelente \\
\hline 5 & Capital Humano & Regular & Deficiente & Bueno \\
\hline 6 & Infraestructura & Bueno & Bueno & Bueno \\
\hline 7 & I+D & Deficiente & Deficiente & Bueno \\
\hline 8 & Cultura Emprendedora & Regular & Deficiente & Excelente \\
\hline
\end{tabular}

\begin{tabular}{|c|c|c|c|c|}
\hline \multicolumn{2}{|c|}{ Determinante } & Costa Rica & México & EE.UU \\
\hline 1 & Financiamiento & 1.00 & 1.67 & 2.33 \\
\hline 2 & Soporte Empresarial & 2.00 & 2.33 & 2.67 \\
\hline 3 & Política & 2.00 & 2.00 & 3.50 \\
\hline 4 & Mercados & 2.00 & 1.83 & 4.00 \\
\hline 5 & Capital Humano & 2.00 & 1.78 & 3.80 \\
\hline 6 & Infraestructura & 3.00 & 3.00 & 3.00 \\
\hline 7 & I+D & 1.75 & 1.50 & 3.33 \\
\hline 8 & Cultura Emprendedora & 2.50 & 1.50 & 4.33 \\
\hline \multicolumn{2}{|r|}{ Promedio } & 2.03 & 1.95 & 3.37 \\
\cline { 2 - 5 } & Estado & Regular & Deficiente & Bueno \\
\cline { 2 - 5 }
\end{tabular}

Figura 7. Resultado global de análisis comparativo Costa Rica, México y EE.UU

Fuente: elaboración propia.

De esta forma se evidencia la brecha de 1,34 puntos entre el EECR regular de Costa Rica y el bueno de Estados Unidos. En cuanto a México, si bien su estado resultó deficiente la diferencia cuantitativa entre este país y Costa Rica es de solo 0,08 puntos. Seguidamente se comentará los resultados por cada determinante.

Financiamiento: El determinante con menor nota para Costa Rica y EE.UU. En el caso de México, no es su peor nota, pero sí resulta con un estado deficiente. En el caso de Costa Rica, todos los componentes dentro de este determinante tienen nota deficiente, una nota de 1,00.

Soporte Empresarial: Este determinante contempla cuatro. En el caso de Costa Rica ninguno supera un estado regular. México concluye también con un estado regular a pesar de tener una nota mejor en 0,3 puntos. Estados Unidos, tampoco cuenta con las mejores condiciones y termina con un estado igual a los otros países.

Política: Otro determinante regular para Costa Rica. Cinco de sus componentes obtienen un el nivel regular. México compensa con una nota buena en el componente de políticas y regulaciones; finalmente obtiene una nota regular para el determinante al igual que Costa Rica. Estados Unidos claramente aventaja a ambos países latinoamericanos, obteniendo una nota buena con una diferencia de 1,5 puntos.

Mercados: Uno de los resultados más dispares entre los componentes internos, ya que Costa Rica tiene notas entre deficientes y buenas, para una nota global regular. México cae a un estado deficiente, a razón de dos componentes deficientes. Mientras Estados Unidos cuenta con una nota global excelente, por lo que es una de las brechas más amplias entre los países latinoamericanos y el norteamericano.

Capital Humano: Este determinante tiene concentrado la mayor cantidad de componentes (9), lo cual ya dice mucho de su importancia en un ecosistema emprendedor. Costa Rica y México obtienen estados regular y deficiente respectivamente. En el caso de Costa Rica, los componentes a priorizar debido a su nota deficiente son: capital humano (en este coincide con México) absorción tecnológica y nivel de emprendimiento en la educación primaria y secundaria.

Infraestructura: Llama la atención debido a que solo un componente de todos los modelos elegidos se logró agrupar en este determinante: el acceso a infraestructura física y servicios. Sin embargo, es un componente que engloba muchos recursos, como son las redes viales, la electricidad, agua e Internet. Un determinante poderoso a la hora de marcar la diferencia para el desarrollo de un emprendimiento y por lo cual se tomó la decisión de analizarlo independientemente. La nota, para los tres países es buena.

Investigación y Desarrollo: Un componente dentro de este determinante que es altamente asociado con la innovación, es la ciencia y tecnología; y es precisamente en el cual Costa Rica puntúa más bajo. Igualmente, 
en el componente de innovar el proceso, este país tiene la mayor brecha con respecto al mismo determinante de Estados Unidos. La nota global es deficiente para Costa Rica y México y buena para Estados Unidos.

Cultura Emprendedora: Este determinante presenta una de las brechas más amplias entre los tres países. En orden, Costa Rica, México y Estados Unidos obtienen un estado regular, deficiente y excelente. La diferencia más alta en puntos está entre México y Estados Unidos (2,83), seguido por Costa Rica y Estados Unidos $(1,83)$, y Costa Rica supera a México por 1 punto de diferencia.

La aceptación al riesgo y el apoyo cultural son los componentes a los que más debe atender Costa Rica; coincidiendo en este último con México.

Finalmente, la figura 8 destaca las brechas a superar para cada par de notas obtenidas en cada determinante, presentando en orden a Costa Rica, México al centro y Estados Unidos.

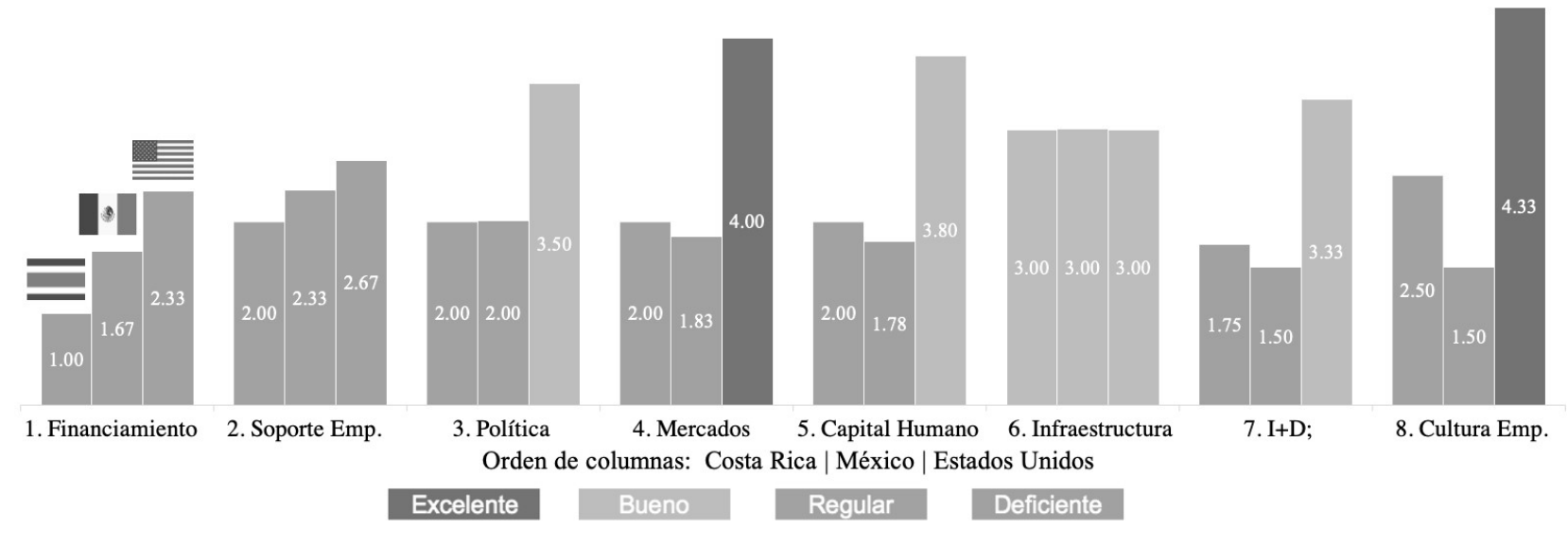

Figura 8. Comparación por determinante: Estados Unidos vs Costa Rica.

Fuente: elaboración propia.

Costa Rica y México logran en su mayoría notas entre deficientes y regulares, solamente consiguen ambos una nota buena en cuanto a Infraestructura. Coinciden también con nota deficiente en Financiamiento e I + D. La diferencia más marcada entre los tres países, es que Estados Unidos no obtiene ninguna nota deficiente y más bien sí dos excelentes.

El análisis especial de los temas de innovación y facilidad de hacer negocios se realiza a razón de la importancia que estos dos ámbitos tienen como parte del ecosistema emprendedor.

Análisis GII 2018: El país de referencia es Suiza, que obtiene el mejor puesto en el ranking 2018. Aún cuando la nota global para Costa Rica es regular $(2,0)$ y para Suiza es buena $(3,7)$, en realidad los dos países varían mucho con respecto al puesto que ocupan (54 para Costa Rica). Suiza no presenta ningún valor deficiente, mientras que sí varios en excelente, cuando para Costa Rica es lo contrario: ningún excelente y varios deficientes.

Análisis Doing Business: El país de referencia es Nueva Zelanda, que obtiene el mejor puesto en el informe 2018. Este modelo es mucho más concentrado, con menos componentes y muy enfocados a su tema central. La excelente noticia es que, de todos los modelos consultados, este es en el cual Costa Rica tiene mejor nota global: 3,8 para un estado bueno. Generando, además, que sea el único modelo de esta investigación en el cual Costa Rica tiene calificaciones excelentes en algún componente, e incluso, el único en el que este país tiene alguna nota mejor que el líder del ranking. Lo anterior refiere al componente conseguir electricidad, donde ambos países tienen un estado de excelente. 


\subsection{Oportunidades de mejora}

Las oportunidades de mejora detectadas para el EECR fueron agrupadas en seis categorías. Seguidamente se detallarán las principales.

Retos de financiamiento y nuevas formas de apoyo: Afinar la capacidad de desarrollar con particular atención "emprendimientos de alto impacto", para que puedan acceder a financiamientos, pero que bajo su condición de crecimiento rápido, rentable y sostenido, puedan retornar los fondos con creces a nivel de la economía (encadenamientos, e inyección de capital por exportaciones).

Sinergia y articulación entre los actores: La especialización de los actores, para que aporten al Ecosistema en puntos específicos de la curva del emprendimiento, brindando una oferta más variada y enfocada a los emprendedores. Esta sinergia permitiría capitalizar esfuerzos bajo una sola visión país, uniendo voluntades, tanto personales como institucionales.

Política: El gobierno central, los locales y sus instituciones, deben buscar dar seguimiento a políticas implementadas por sus predecesores, de manera que se pueda construir sumando, en lugar de "reconstruir" totalmente encima de los esfuerzos anteriores.

Cultura, educación y networking: Estimular la cultura emprendedora en todos los niveles de la educación formal, para que no se pierda la creatividad innata que tienen los niños para buscar soluciones innovadoras y disruptivas a los problemas. Además, allanar el camino estratégicamente para que exista menor miedo a emprender y mejores actitudes para darle seguimiento exitoso a un emprendimiento.

Vacíos de mercado, sostenibilidad e innovación: Se deberían considerar los Objetivos de Desarrollo Sostenible (ODS) de Naciones Unidas como una oportunidad de tomar una o varias de estas banderas y comprometerse con ellas. Se pueden identificar vacíos de mercado y aprovechar el espíritu emprendedor para dar soluciones.

Alcance nacional: Es indiscutible que Costa Rica es territorialmente pequeña, contando con acceso rápido hacia sus diferentes extremos, y buen servicio de telecomunicaciones. Esta realidad debe tenerla presente la comunidad de actores, para unir esfuerzos en descentralizar las acciones del Ecosistema de la Gran Área Metropolitana (GAM).

\section{Conclusiones}

Este estudio inició con el objetivo de evaluar el estado del ecosistema emprendedor en Costa Rica al segundo semestre de 2018, así como sus oportunidades de mejora. La evaluación puntualmente concluye que el estado del Ecosistema Emprendedor de Costa Rica es regular. Los determinantes de Financiamiento e Investigación \& Desarrollo son los peor calificados (deficientes), solamente Infraestructura tiene una condición buena y los cinco restantes son regulares (Soporte Empresarial, Política, Mercados, Capital Humano, Infraestructura y Cultura Emprendedora).

La metodología, basada en el octeto de determinantes ANDE, el diseño de la matriz para la integración de las variables y la construcción de una robusta herramienta de evaluación diseñada en Excel, puede ser repetida en un futuro cercano para dar seguimiento a los resultados e incluso puede replicarse en cualquier país que tenga la información requerida.

Como cualquier estudio, el nuestro no está exento de limitaciones. Destacan dos. La ausencia de evaluaciones nacionales GEM para Costa Rica posterior a 2014 así como las diferentes fechas de emisión de los informes internacionales, que al momento de realizar la investigación generó la necesidad de trabajar algunas ediciones 2017 y otras 2018.

Algunas futuras líneas de investigación que emanan de este artículo son evaluar ecosistemas de emprendi- 
miento regionales dentro del territorio nacional, para comprender las diferencias, sus circunstancias y necesidades. Asimismo, realizar estudios independientes sobre cada uno de los ocho determinantes del Ecosistema, de modo que se pueda profundizar mucho más tanto en la evaluación, sus actores, como en las propuestas.

\section{Referencias}

[1] Academy of Management. (s.f.). History. Recuperado el 9 de agosto de 2018, de http://aom.org/AboutAOM/History.aspx

[2] Ács, Z. J., Szerb, L. \& Lloyd, A. (2018). Global Entrepreneurship Index 2018. Recuperado de https://thegedi.org/

[3] Aspen Network of Development Entrepreneurs. (2013). Entrepreneurial Ecosystem Diagnostic Tookit. Recuperado de https://www.andeglobal.org/

[4] Aspen Network of Development Entrepreneurs. (s.f.). About ANDE. Recuperado el 16 de agosto de https://www.andeglobal.org

[5] Audretsch, D. B., \& Belitski, M. (2016). Entrepreneurial ecosystems in cities: Establishing the framework conditions. The Journal of Technology Transfer, 42(5), 1030-1051. https://doi.org/10.1007/s10961-016-9473-8

[6] Auerswald, P. (2015). Enabling entrepreneurial ecosystems. In D. Audretsch, A. Link, \& M. L. Walsok (Eds.), The Oxford handbook of local competitiveness (pp. 54-83). Oxford, U.K.: Oxford University Press. https://doi.org/10.1093/oxfordhb/9780199993307.013.4

[7] Babson Entrepreneurship Ecosystem Project. (2015). Entrepreneurship Ecosystem Lessons. Recuperado el 16 de agosto de http://entrepreneurial-revolution.com/

[8] Brown, R., \& Mason, C. (2017). Looking inside the spiky bits: a critical review and conceptualisation of entrepreneurial ecosystems. Small Business Economics, 49(1), 11-30. https://doi:10.1007/s11187-017-9865-7

[9] Cornell University, INSEAD \& World Intellectual Property Organization. (2018). The Global Innovation Index 2018: Energizing the World with Innovation. Recuperado de https://www.globalinnovationindex.org/

[10] Feld, B. (2012). Startup communities: Building an entrepreneurial ecosystem in your city. Wiley. https://doi.org/10.1002/9781119204459

[11] Global Entrepreneurship Research Association. (2018). Global Entrepreneurship Monitor: Global Report 2017/18. Recuperado de https://www.gemconsortium.org/report/50012

[12] Global University Entrepreneurial Spirit Students Survey. (2017). Key facts for the GUESS project. Recuperado de http://www.guesssurvey.org/keyfacts/

[13] Isenberg, D. (2010). How to Start an Entrepreneurial Revolution. Harvard Business Review, 88(6), 40-50.

[14] Kantis, H. (2011, junio 7). ¿Quién enseña a quién? Recuperado de https://prodem.ungs.edu.ar/quien-ensena-aquien/

[15] Kantis, H., Federico, J. \& Ibarra García, S. (2017). Condiciones Sistémicas para el Emprendimiento Dinámico 2017- América Latina: avances y retrocesos en perspectiva. Recuperado de https://prodem.ungs.edu.ar/

[16] Lebendiker, M., Herrera, R. \& Velásquez, G. (2015). Reporte Nacional 2014: la situación del emprendimiento en Costa Rica, una perspectiva local sobre emprendimientos, retos y crecimientos en Costa Rica. Recuperado de https://www.gemconsortium.org/

[17] McKenzie B.M. \& Sud M. (2009). Prolegomena to a new ecological perspective in entrepreneurship. Academy of Entrepreneurship Journal, 15(1), 43-60

[18] Mason, C. M., \& Brown, R. (2014). Entrepreneurial ecosystems and growth oriented entrepreneurship. The Hague, Netherlands: OECD LEED Program.

[19] Ministerio de Economía Industria y Comercio de Costa Rica. (2010). Costa Rica Emprende: Política Nacional de Emprendimiento Administración Chinchilla-Miranda 2010-2014. Recuperado de http://www.pyme.go.cr/media/archivo/normativas/PoliticaNacEmpren.pdf 
[20] Ministerio de Economía Industria y Comercio de Costa Rica. (2014). Política de Fomento al Emprendimiento de Costa Rica, 2014-2018. Recuperado de http://www.pyme.go.cr/media/archivo/normativas/politicafomentoalemprendimiento.pdf

[21] Ministerio de Economía Industria y Comercio de Costa Rica. (2020). Política Nacional de Empresariedad 2030. Recuperado de http://reventazon.meic.go.cr/informacion/pyme/MEIC_PNE_2030.pdf

[22] Ministerio de Planificación Nacional y Política Económica (2018). Consulta Ciudadana: Plan de Desarrollo y de Inversión Pública 2019-2022. Recuperado de https://sites.google.com/expedientesmideplan.go.cr/pndip/inicio

[23] Minniti, M. (2012). El Emprendimiento y el Crecimiento Económico de las Naciones. Economía Industrial, 383, 23-30.

[24] O'Connor A., Stam E., Sussan F., Audretsch D.B. (2018). Entrepreneurial Ecosystems: The Foundations of Place-based Renewal. En O'Connor A., Stam E., Sussan F., Audretsch D. (Eds). Entrepreneurial ecosystems: Place-based transformations and transitions, 1-21. Springer. https://doi.org/10.1007/978-3-319-63531-6_1

[25] Qian, H., Acs, Z.J., \& Stough, R.R. (2013). Regional systems of entrepreneurship: The nexus of human capital, knowledge and new firm formation. Journal of Economic Geography, 13(4), 559-587. https://doi.org/10.1093/ jeg/lbs009

[26] Spigel, B. (2017). The relational organization of entrepreneurial ecosystems. Entrepreneurship Theory and Practice, 41(1), 49-72. https://doi.org/10.1111/etap.12167

[27] Stam E. (2018). Measuring entrepreneurial ecosystems. En A. O'Connor, E. Stam, F. Sussan,y D. Audretsch (Eds.), Entrepreneurial ecosystems: Place-based transformations and transitions, 173-197, Springer. https://doi.org/10.1515/zfw-2019-0001

[28] Stam, E. (2015). Entrepreneurial ecosystems and regional policy: a sympathetic critique. European Planning Studies, 23(9), 1759-1769. https://doi.org/10.1080/09654313.2015.1061484

[29] Szerb, L., Lafuente, E., Horváth, K., Páger, B. (2018). The relevance of quantity and quality entrepreneurship for regional performance: The moderating role of the entrepreneurial ecosystem. Regional Studies, 53 (9), 1308-1320. https://doi.org/10.1080/00343404.2018.1510481

[30] World Bank Group (2018). Doing Business 2018: Reforming to Create Jobs. Recuperado de http://www.doingbusiness.org/ 